\title{
Terrorist Threat Mitigation Strategies for the Railways
}

\author{
Abigail Luxton ${ }^{1}$ and Marin Marinov ${ }^{2, *}$ (1) \\ 1 School of Mechanical and Systems Engineering, Newcastle University, Newcastle upon Tyne NE1 7RU, UK \\ 2 Engineering Systems and Management (ESM), School of Engineering and Applied Science (EAS), Aston \\ University, Birmingham B4 7ET, UK \\ * Correspondence: m.marinov@aston.ac.uk
}

Received: 18 March 2020; Accepted: 15 April 2020; Published: 22 April 2020

\begin{abstract}
Terrorism is an ever-present threat that is becoming more prevalent in today's society. The railway has always been a favourable target for terrorists due to the high volume of people that utilise it, as well as the vital services it provides to the nation. The railway is expanding and this will likely result in it becoming increasingly vulnerable to attack, unless new mitigation solutions are devised. In this paper risk assessment analyses by a software package conducted on different sections of the railway station provided insight into the present vulnerabilities. Mitigation solutions were devised to abolish these weaknesses in the system, to avoid terrorist exploitation. They were then evaluated to establish a workable solution: a modified version of the Thales Smart Corridor. This workable solution should be implemented across the nation to ensure passenger and rail infrastructure protection.
\end{abstract}

Keywords: terrorist attacks; railways; risk assessment; analysis; terrorist threat mitigation strategies

\section{Introduction}

September 11th is a date forever ingrained in the memories of the world as one of the worst aterrorist attacks in modern history. With 2996 dead in a day, [1], the incident highlighted the global requirement to re-evaluate how safety is ensured. Global terrorism is becoming increasingly common, with approximately 700 incidents in 1970 rising to near 16,800 incidents in 2014 alone [2]. Stories of conflict and destruction fill our newspapers, the latest of which in Europe, occurred on 22 March 2017 on Westminster Bridge in London [3]. Attacks of terror take many forms. Although their purposes are often unknown, they incite fear and are typically used to demonstrate rebellion against an aspect of a civilisation they disagree with. A recurrent target of these attacks is popular infrastructure, with establishments such as airports and train stations being prime targets, due to the heavy foot traffic, the 'open and accessible designs' of railway stations [4], and vital services they provide to society. Airports have rigorous security checks and extensive safety protocols. However, railway stations do not have such procedures in place, although a study conducted by Professor Arnold Barnett, the George Eastman Professor of Management Science and a Professor of Statistics at the MIT Sloan School of Management [5], found the risk per mile of rail travel is ten times greater than if you were to travel by air [6]. Yet the railway stations rely on CCTV, public vigilance, and the occasional security guard to maintain the peace. Despite the acknowledgement from Adrian Dwyer, the British Transport Police's counter terrorism risk advisor, that 'ensuring anything like $100 \%$ protection against terrorist attack is not a viable option' [7], the current means of terrorism mitigation are insufficient in providing consistently assured public safety. The 2004 attack on the Metro in Madrid demonstrated just how vulnerable the railway currently is, with 191 dead and 1841 wounded [8]. Terrorism is on the rise and the railways are expanding. Due to the developments in high speed rail technology [9-11], baggage handling systems [12-14], and urban freight by rail [15-17], the railway network is set to expand [18]. 
This expansion will make rail travel a greater target for terrorist activities. Alternate, effective terrorist threat mitigation strategies must be devised to ensure the sustained protection of the public and railway infrastructure.

\section{Objective, Methodology, and Scope}

The objective of this study is to identify the prevalent risks/threats to railway, metro, and urban passenger freight, and propose a workable solution to help mitigate these risks/threats and to most effectively implement it.

This has been achieved through the following stepwise methodology:

1. Identify the potential risks and threats to people and infrastructure.

2. Establish where these risks and threats have the most devastating impact.

3. Provide mitigation strategies for these risks and threats.

4. Evaluate the mitigation strategies used to obtain a workable solution.

5. Determine how this workable solution is to be implemented.

A comprehensive data analysis, 1972-2011, is conducted in [19] to better understand the evolution of terrorist attacks targeting rail and metro systems. The data analysis looked at geographical distribution of terrorist attacks and drew conclusions about the most targeted elements and components of the railway system (rail lines followed by trains and stations) [20,21]. It was found that the most used tool for terrorist act is a bomb carried by a suicide bomber. When referring to the rail and metro stations they concluded that the medium and small size stations are more vulnerable and easier to attack. As for countermeasures and tools, the most common are visual cameras, though they are not the most effective. It was concluded that the most effective tools to prevent terrorist attempts are the security guards. Although every station is characterised by certain peculiarities $[9,12,13,20,21]$ it was concluded that a more innovative security approach with a better understanding of the rail operation pattern behing can have a positive effect on every rail station vulnerability level and railway systems as a whole.

According to [22], technical systems employed presently in metros cannot prevent bringing explosive devices of a certain type into the metro. This is quite worrying and presents us with an area of concern and certainly requires special attention. [23] organised 20 interviews with experts and concluded that there are certain problems when tackling vulnerabilities in preparedness, which result in an inability to ensure that trains are secure when acts of antagonistic violence occur. This outlines another area of concern when it comes to terrorist attempts to cause severe damages to the railway facilities and systems. In addition, [11], after having analysed the capabilities of technical security systems applied to protect high-speed (HS) trains, concluded that there is no protection against terrorist attacks using drones that carry bombs and explosives. To resolve this threat, suppression by radio interference generated by a national security system is proposed to interfere, devalue, neutralise, and remove the harmful device. But the efficiency of this suggestion depends on many factors, very first of which is how reliable the national security system is.

For measures to observe and protect railway networks in urban areas in particular, [24] developed a game theory model for scheduling professional teams patrolling an urban railway network. They set up a non-cooperative simultaneous move game between a defender and an attacker and obtained promising results for patrol scheduling of a real-world urban railway network. Although patrols are crucial for defending and protecting the railways, setting up an efficient and most workable schedule is a huge challenge. Because patrol schedules have a repetitive nature, terrorists can easily fool the patrols, distract them, and do their malicious damage. Patrolling requires precision, a lot of resources, and 24/7 human presence. Because we live in a digital era, it is believed that digitalisation and new technology for surveillance, object detection, and recognition will provide new and more efficient means for us to observe and protect the railways. 
In [25], is argued that there are not any published documents on a simplified approach to the risk assessment and protection of existing subway stations, which is rather worrying if we consider the fact that protecting the railways is supposed to be highly confidential. Nevertheless, a Threat and Vulnerability Risk Assessment (TVRA) applied to a generic subway station was developed and published to advise on mitigation strategies expected to mitigate the risk of terrorist attempts and malicious acts intended to damage existing subway stations. [26] developed a risk-based decision-making framework on a basis of probabilistic risk assessment (PRA). Their work was inspired by [27] and proposed that the state of partial neutralization affects the decisions of the attacker and yields different loss scenarios. The framework proposed need to be revisited and updated when more information becomes available, though. Also a better use of existing knowledge and technology for utility coherence, layered security systems, data fusion, and possibly implementation of wireless sensors needs to be considered, ensured, and demonstrated for better response to malicious terrorist attempts to be identified, captured, and prevented.

This paper explores the current nature of terrorism and its exploitation of public rail transport systems by recognising the particular interest in the railways whilst examining existing railway terrorism avoidance and prevention mechanisms, describing them as insufficient. It then proceeds to identify the aims of the paper, which are to establish the potential risks to people and infrastructure when considering terrorism on the railways, including where these risks would have the most devastating impact, mitigation strategies, an evaluation to obtain a workable solution, and how the solution should be implemented. Risk assessments are then conducted on each area of a station to thoroughly investigate the risks and threats that specific regions would be vulnerable to, in order to then devise appropriate mitigation solutions; this is done with the Risk Manager application software. The reason for the selection of this software is then discussed. Risk assessments are conducted for the station platforms, trains, tunnels/walkways, shops/restaurants, waiting rooms, and the atrium. The shops and restaurants are identified as the locations with the greatest associated risk. The mitigation strategies used in the risk assessments are then evaluated. Calculations to analyse the probability of a terrorist attack occurring on the railway in the next five years are then conducted, with the resultant likelihood of an attack being $91 \%$. The paper subsequently investigates the impact a mitigation solution would have on population flow and the cost benefit. Then it considers the proposed solution to search both passengers and their luggage, potentially with a modified Thales Smart Corridor. The triggers that cause terrorists to act are examined as potential means of prevention as they are the key risks that lead to the eradication of all linked risks. It then analyses the elements of a workable solution, including dispelling any concerns, describing the solution necessity and installation considerations. It then describes how to further the paper's research in the future.

\section{Risk Assessment Software}

In order to fully investigate the risks and threats that train stations are vulnerable to, it is necessary to divide the station into sections and apply individual risk assessments to them. This is due to the fact that vulnerability to attack varies across different parts of the station as a result of the nature of the infrastructure in specific locations. A number of risk assessment methodologies exist that analyse the particular risks associated with the railway. These include the RAMPART methodology, directed at metro and light-rail services [28]. This methodology was modified and produced the SecuRail toolbox. The SecuRail toolbox is unique in that it 'incorporates counter measures as well as cost benefit analysis' [29]. However, the use of these applications was dismissed in favour of the generation of a more comprehensive risk assessment, which would allow for the production of a universal mitigation strategy that could be applied to various types of rail, not exclusively metro and light rail.

This study explores the use of the Risk Manager application software to examine different areas of train stations and their infrastructure through individual location risk assessments. The purpose of this software and how it is utilised serve to evaluate the effectiveness of mitigation solutions on a rudimentary basis. The software allows for numerous risks to be input into a single database (Figure 1). 
Within individual edit windows, the properties of each risk are then defined more extensively, including the risk identification, the pre-mitigation assessment, the risk mitigation and post-mitigation assessment details (Figure 2). Outcomes of the assessments are displayed in charts depicting the probability of attacks colour-coded according to increasing severity risk, should it occur, and displaying the percentage likelihood that an attack of this severity would occur. The mitigation strategies that could be adopted to lessen or eliminate the possibility of attack are then theoretically utilised, and the revised assessment charts are generated to provide a visual comparison and percentage analysis of residual risk severity after the mitigation solutions have been applied to assess their effectiveness.

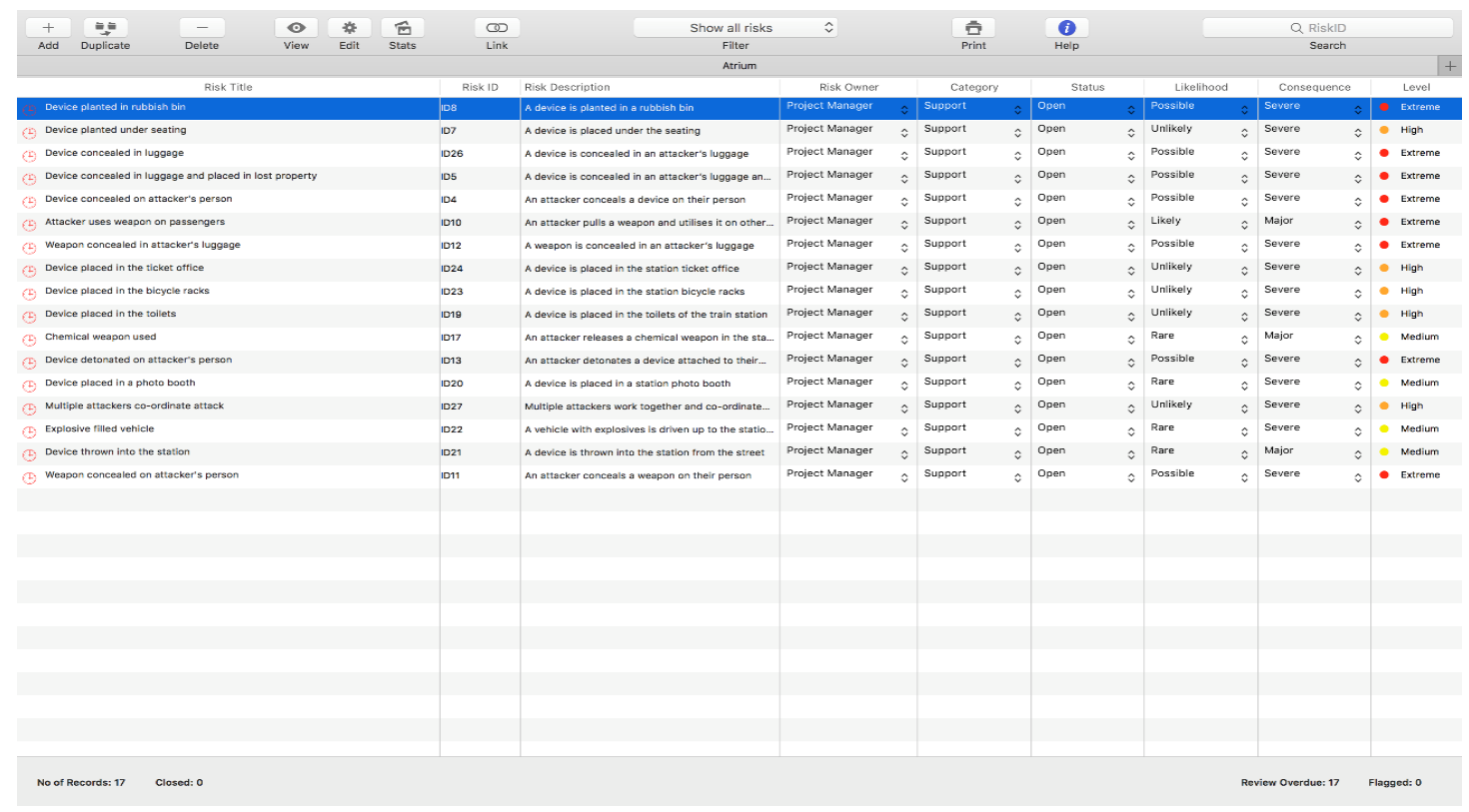

Figure 1. Screenshot of Risk Manager application analysis of the Atrium.

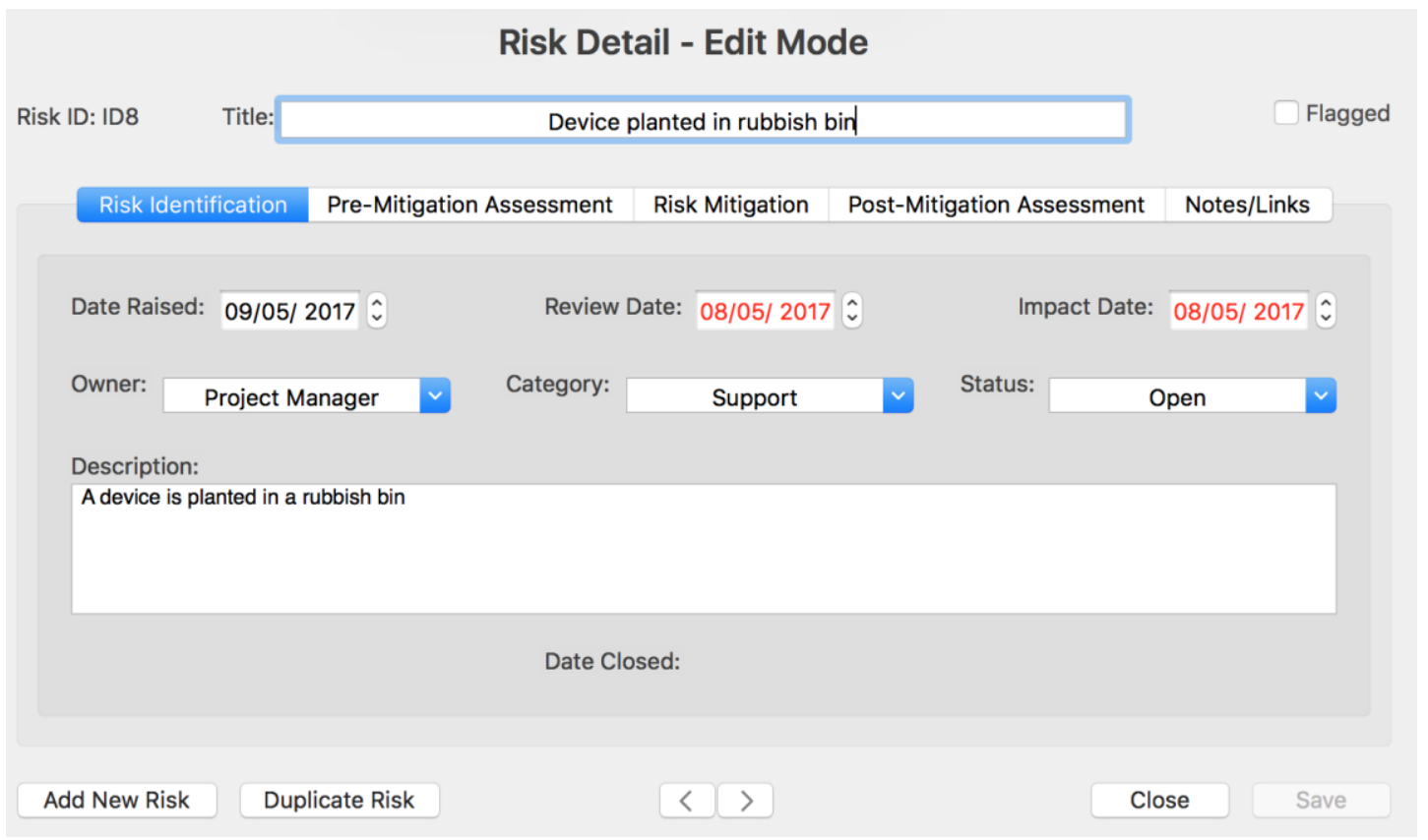

Figure 2. Screenshot of a Risk Manager individual risk edit window. 


\section{Risk Assessments}

\subsection{Platforms}

Platforms are the areas in train stations where commuters wait to board their trains, and they are usually equipped with seating, rubbish bins, and occasionally miniature cafes serving light refreshments. Small offices may also be located on station platforms. Risks and mitigation solutions for platforms are provided in Table 1.

Table 1. Risks and mitigation solutions for platforms.

\begin{tabular}{cc}
\hline Identified Risks & Mitigation Solutions \\
\hline Passenger pushed onto tracks & Prevent access to the tracks \\
Device planted in rubbish bin & 24/7 CCTV \\
Device planted under platform seating & Security guards on the platform \\
Device planted in platform offices & Search passenger bags \\
Device concealed in luggage & Search passengers \\
Device concealed on attacker's person & Sniffer dogs on the platform \\
Knife obtained at a station shop & Remove rubbish bins from the platform \\
Device planted in platform cafe & Only permit staff access to platform offices \\
Attacker uses weapon on passengers & Eliminate openings on the office front, speak through windows to passengers \\
Weapon concealed in attacker's luggage & Device detection system on the platform \\
Device detonated on attacker's person & Do not stock knives at station shop \\
Weapon concealed on attacker's person & Lock cafe at night \\
Device planted on tracks & Man cafe at all times \\
\hline
\end{tabular}

Chart 1 depicts that the platforms have extreme, high, and low risks associated with them. The chance of an extremely severe risk occurring is 53.8\%, a highly severe risk occurring is $30.8 \%$, and there is a $15.4 \%$ chance that a low severity risk will occur.

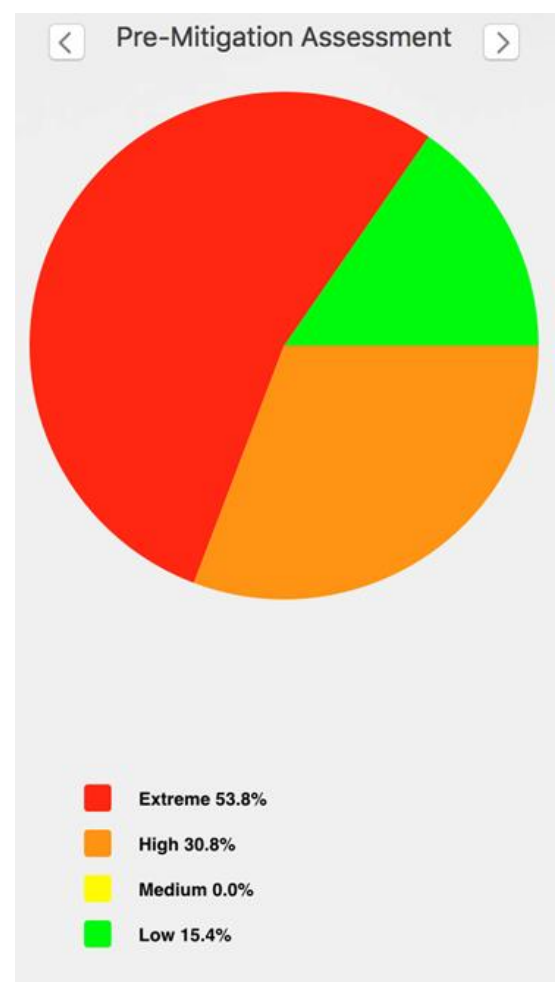

Chart 1. Platform risk severity pre-mitigation assessment.

Chart 2 depicts that after the mitigation solutions have theoretically been applied, the platforms have high, medium, and low risks associated with them. The chance of a highly severe risk occurring 
is $53.8 \%$, a medium severity risk occurring is $30.8 \%$, and there is still a $15.4 \%$ chance that a low severity risk will occur.

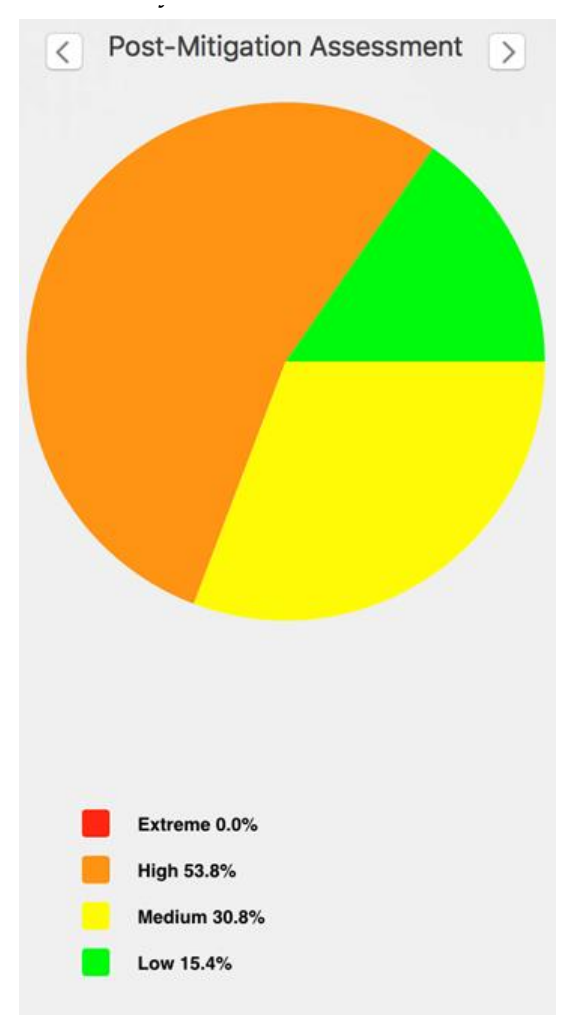

Chart 2. Platform risk severity post-mitigation assessment.

\subsection{Trains}

The trains themselves are at risk as millions of passengers use them, yet when in use their escape routes are rendered practically non-existent. Trains completely confine passengers, potentially for hours, until the next station. Risks and mitigation solutions for trains are given in Table 2.

Table 2. Risks and mitigation solutions for trains.

\begin{tabular}{cc}
\hline Identified Risks. & Mitigation Solutions \\
\hline Device planted in the toilets & Device detection system in the toilets \\
Device planted in rubbish bin & 24/7 CCTV on trains \\
Device planted under seating & Security guards in the station \\
Device planted in luggage racks & Search passenger bags pre boarding \\
Device concealed in luggage & Search passengers pre boarding \\
Device concealed on attacker's person & Sniffer dogs in the station \\
Chemical weapon used & Remove rubbish bins from train \\
Device planted in platform cafe & Fire extinguishers on the train \\
Attacker uses weapon on passengers & Smoke detectors across the train \\
Weapon concealed in attacker's luggage & Use flame-retardant material for upholstery \\
Device detonated on attacker's person & \\
Weapon concealed on attacker's person & \\
Attacker starts a fire with upholstery & \\
\hline
\end{tabular}

Chart 3 depicts that the trains have extreme and medium risks associated with them. The chance of an extremely severe risk occurring is $87.5 \%$, and there is a $12.5 \%$ chance that a medium severity risk will occur. 


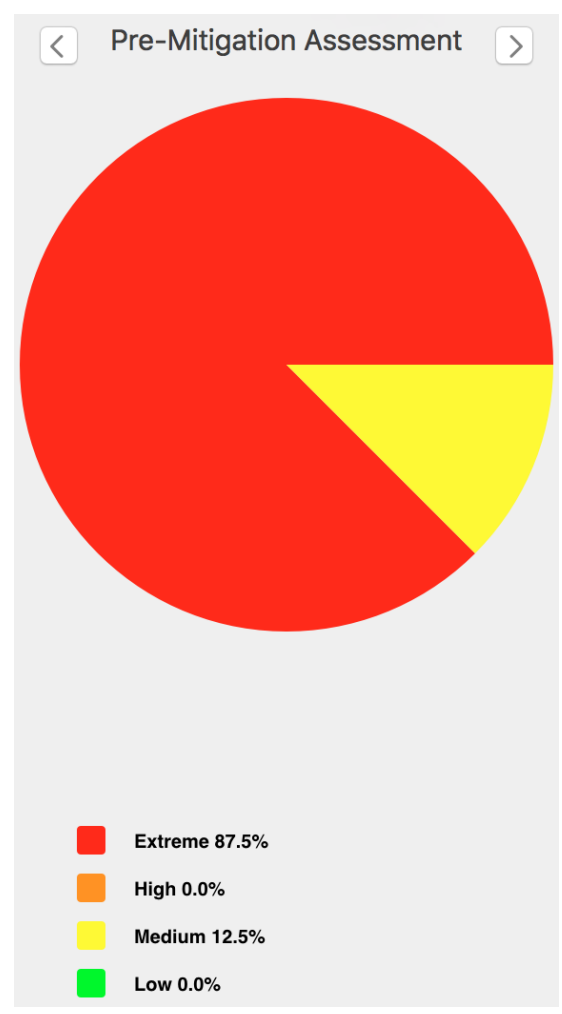

Chart 3. Train risk severity pre-mitigation assessment.

Chart 4 depicts that after the mitigation solutions have theoretically been applied, the trains have high and medium risks associated with them. The chance of a highly severe risk occurring is $75 \%$, and there is still a $25 \%$ chance that a medium severity risk will occur.

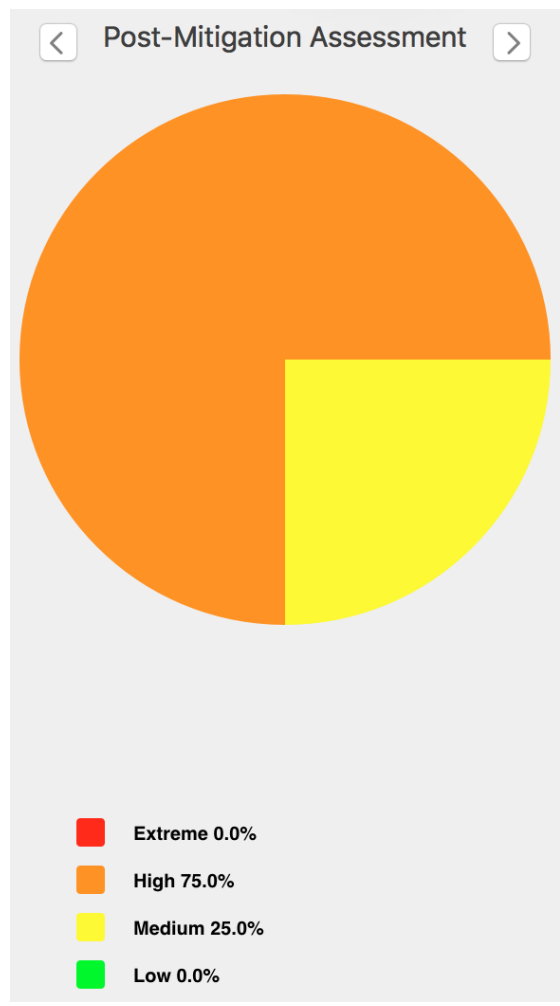

Chart 4 . Train risk severity post-mitigation assessment. 


\subsection{Tunnels/Walkways}

Tunnels and walkways provide the access routes from the ticket hall to the shops/restaurants or the platforms. They are sometimes fully enclosed and occasionally just corridors. They experience a considerable amount of foot traffic from passengers either boarding or alighting from trains, in potentially restricted or confined spaces. Risks and mitigation solutions for tunnels/walkways are presented in Table 3.

Table 3. Risks and mitigation solutions for tunnels/walkways.

\begin{tabular}{cc}
\hline Identified Risks & Mitigation Solutions \\
\hline Device planted in rubbish bin & $24 / 7$ CCTV \\
Chemical weapon used & Security guards in the station \\
Attacker uses weapon on passengers & Search passenger bags \\
Device concealed in luggage & Search passengers \\
Device concealed on attacker's person & Sniffer dogs in the station \\
Weapon concealed in attacker's luggage & Remove rubbish bins from the platform \\
Weapon concealed on attacker's person & \\
Device detonated on attacker's person & \\
\hline
\end{tabular}

Chart 5 depicts that the tunnels/walkways have extreme, high, medium, and low risks associated with them. The chance of an extremely severe risk occurring is $66.7 \%$, a highly severe risk occurring is $16.7 \%$, a medium severity risk is $8.3 \%$, and a there is an $8.3 \%$ chance that a low severity risk will occur.

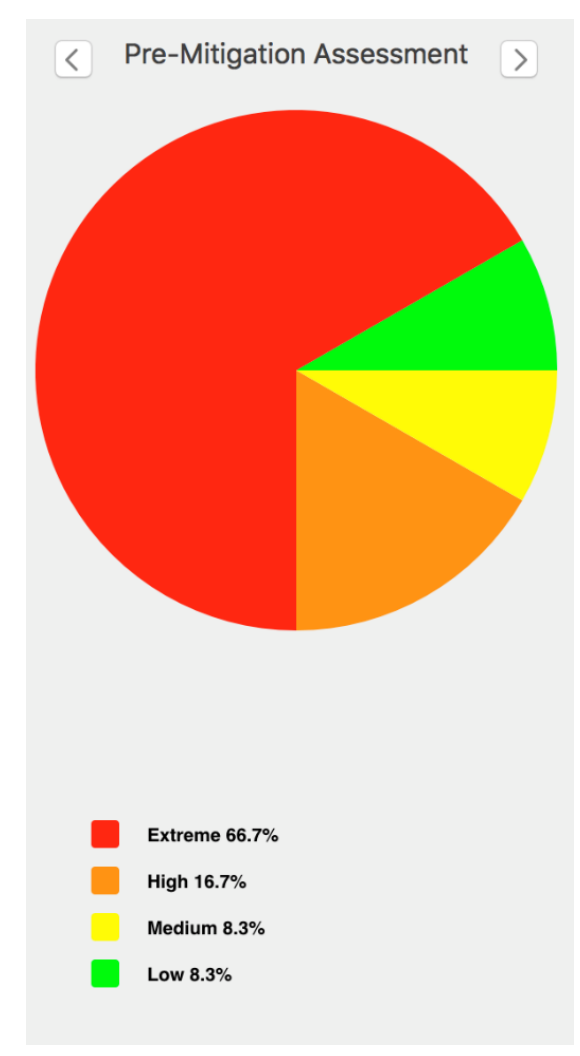

Chart 5. Tunnels/Walkways risk severity pre-mitigation assessment.

Chart 6 depicts that after the mitigation solutions have theoretically been applied, the tunnels/walkways have high, medium, and low risks associated with them. The chance of a highly severe risk occurring is $66.7 \%$, a medium severity risk occurring is $25 \%$, and there is still an $8.3 \%$ chance that a low severity risk will occur. 


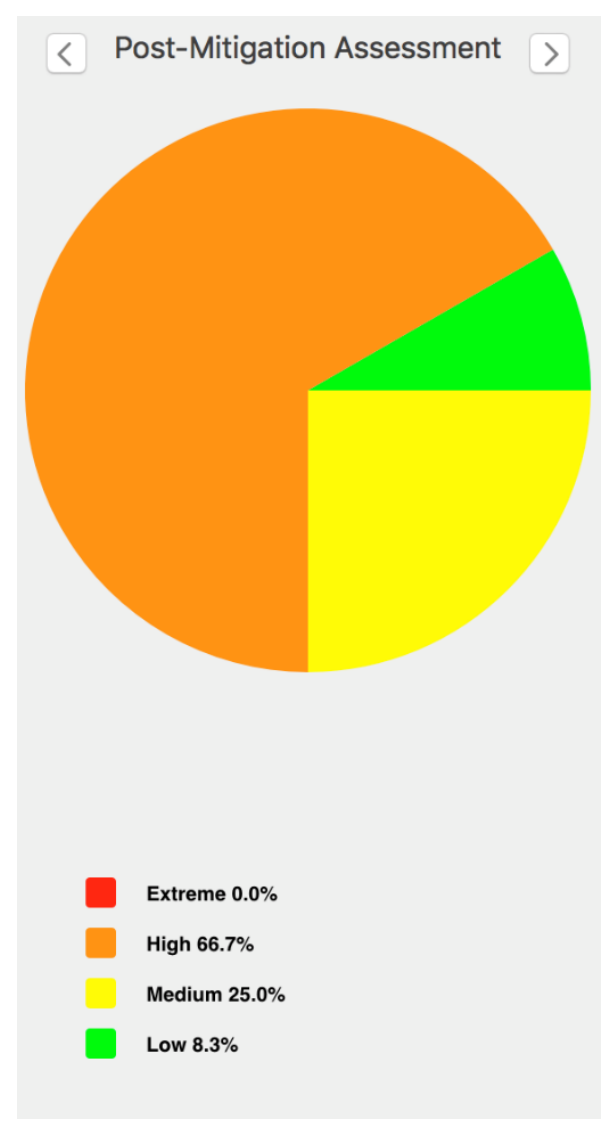

Chart 6. Tunnels/Walkways risk severity post-mitigation assessment.

\subsection{Shops/Restaurants}

The station shops and restaurants, although usually connected to the atrium, have minimal entrances and exits (usually just one). Due to their contents, shops and restaurants provide more secluded and less conspicuous areas to place devices unnoticed. Risks and mitigation solutions for shops/restaurants are given in Table 4.

Table 4. Risks and mitigation solutions for shops/restaurants.

\begin{tabular}{cc}
\hline Identified Risks & Mitigation Solutions \\
\hline Device planted in rubbish bin & 24/7 CCTV \\
Device planted under seating & Security guards in the station \\
Device planted in the shop/restaurant toilets & Search passenger bags \\
Device concealed in luggage & Search passengers \\
Device concealed on attacker's person & Sniffer dogs in the station \\
Chemical weapon used & Remove rubbish bins with public access \\
Attacker starts a fire with upholstery & Device detection system in the toilets \\
Attacker uses weapon on passengers & Use flame-retardant material on restaurant seating \\
Weapon concealed in attacker's luggage & Smoke detectors in the shop/restaurant \\
Device detonated on attacker's person & Fire extinguishers in the shop/restaurant \\
Weapon concealed on attacker's person & Only permit staff access to cooking equipment \\
Utensils in restaurant utilised as weapons & Provide plastic utensils instead of metal \\
Merchandise in shop utilised as a weapon & Security tag all merchandise \\
& Keep all merchandise that could be weaponised in a locked case \\
\hline
\end{tabular}

Chart 7 depicts that the shops/restaurants have extreme, high, medium, and low risks associated with them. The chance of an extremely severe risk occurring is $63.6 \%$, a highly severe risk occurring is $18.2 \%$, a medium risk is $9.1 \%$, and there is a $9.1 \%$ chance that a low severity risk will occur. 


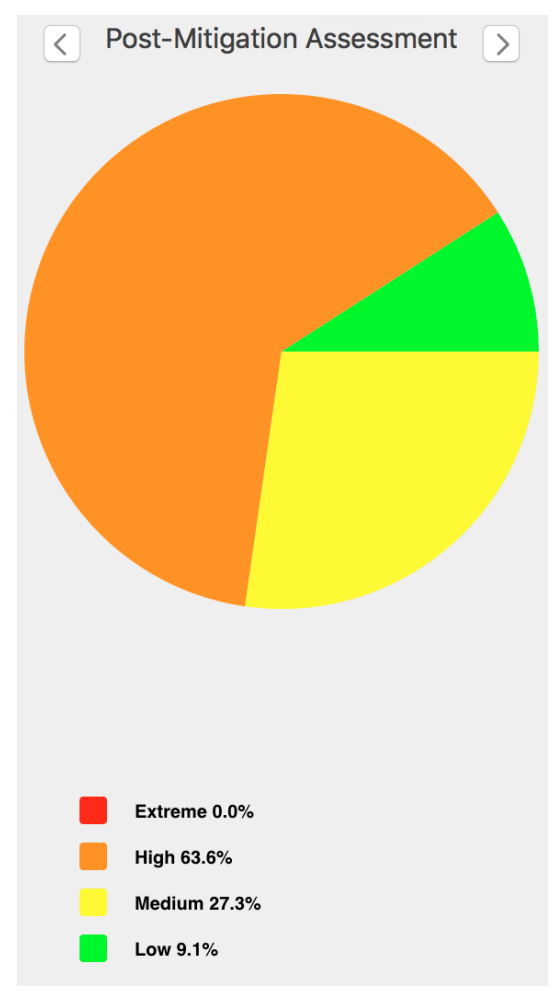

Chart 7. Shops/restaurants risk severity pre-mitigation assessment.

Chart 8 depicts that after the mitigation solutions have been theoretically applied shops/restaurants have high, medium, and low risks associated with them. The chance of a highly severe risk occurring is $63.6 \%$, a medium severity risk occurring is $27.3 \%$, and there is still a $9.1 \%$ chance that a low severity risk will occur.

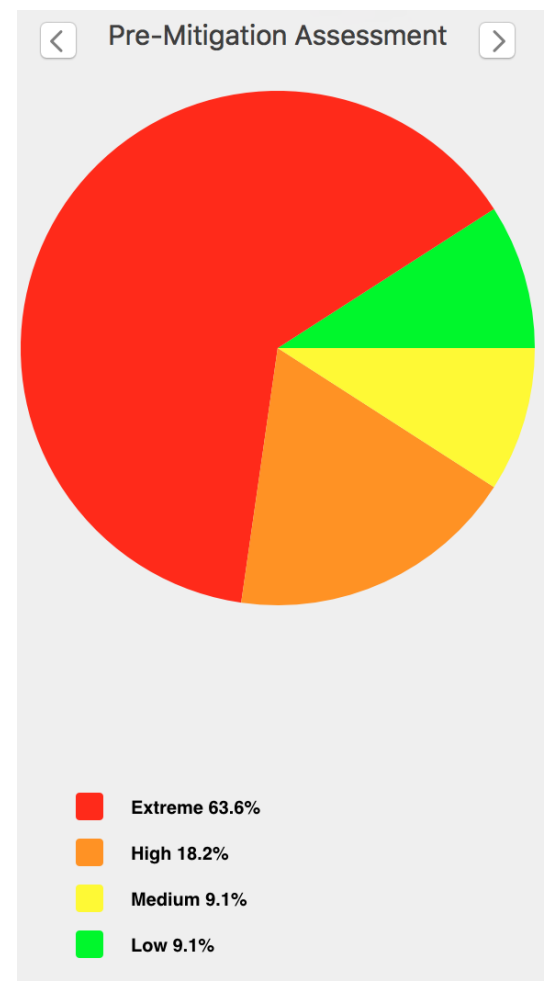

Chart 8. Shops/restaurants risk severity post-mitigation assessment. 


\subsection{Waiting Rooms}

Waiting rooms across the station are where passengers await their trains. Similar to shops and restaurants, although they are not significantly more accessible, they still provide cover for potentially unscrupulous activity. Risks and mitigation solutions for waiting rooms are presented in Table 5.

Table 5. Risks and mitigation solutions for waiting rooms.

\begin{tabular}{cc}
\hline Identified Risks & Mitigation Solutions \\
\hline Device planted in rubbish bin & 24/7 CCTV \\
Device planted under seating & Security guards in the station \\
Device placed in waiting room toilets & Search passenger bags \\
Device concealed in luggage & Search passengers \\
Device concealed on attacker's person & Sniffer dogs in the station \\
Chemical weapon used & Remove rubbish bins from waiting rooms \\
Attacker starts a fire with upholstery & Device detection system in the toilets \\
Attacker uses weapon on passengers & Use flame-retardant material on the seating \\
Weapon concealed in attacker's luggage & Smoke detectors in the waiting rooms \\
Device detonated on attacker's person & Fire extinguishers in the waiting rooms \\
Weapon concealed on attacker's person & \\
\hline
\end{tabular}

Chart 9 depicts that the waiting rooms have extreme, high, medium, and low risks associated with them. The chance of an extremely severe risk occurring is $53.8 \%$, a highly severe risk occurring is $15.4 \%$, a medium risk is $23.1 \%$, and there is a $7.7 \%$ chance that a low severity risk will occur.

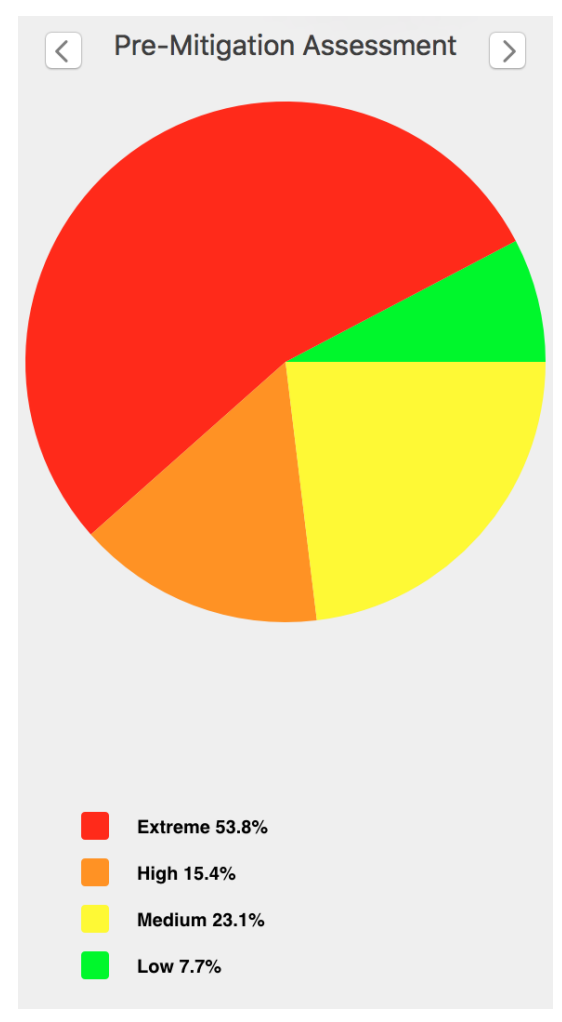

Chart 9. Waiting rooms risk severity pre-mitigation assessment.

Chart 10 depicts that after the mitigation solutions have been theoretically applied the waiting rooms have high, medium, and low risks associated with them. The chance of a highly severe risk occurring is $53.8 \%$, a medium severity risk occurring is $23.1 \%$, and there is still a $23.1 \%$ chance that a low severity risk will occur. 


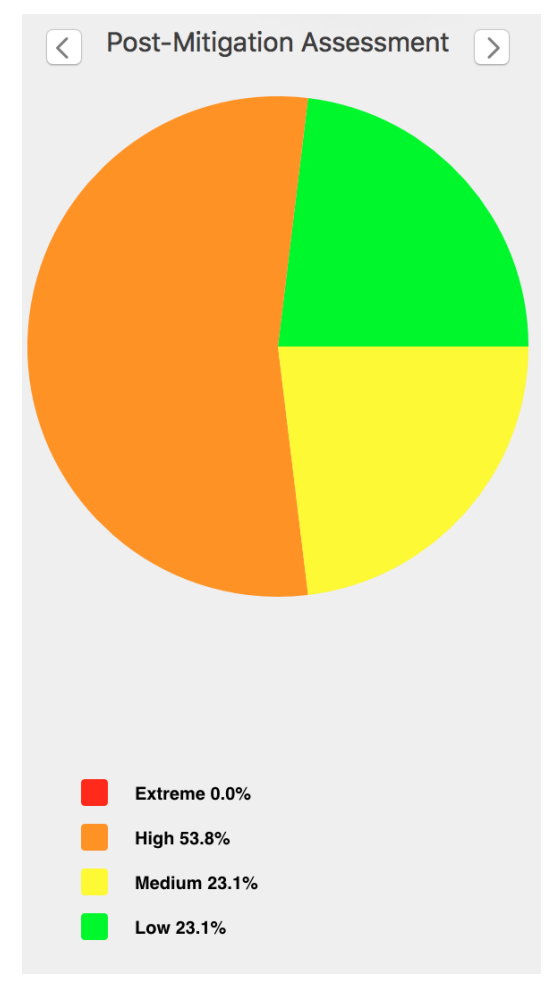

Chart 10. Waiting rooms risk severity post-mitigation assessment.

\subsection{Atrium}

The atrium is the open area in all medium-to-large stations occasionally referred to as the Concourse or Ticket Hall. It displays train and platform information, contains the ticket offices and the toilets, as well as many other features of the train station. It incorporates shops and restaurants, should the station have any, as well as multiple enquiry offices. The atrium will often be at street level and consist of multiple entrances and exits. Risks and mitigation solutions for atriums are provided in Table 6.

Table 6. Risks and mitigation solutions for atriums.

\begin{tabular}{cc}
\hline Identified Risks & Mitigation Solutions \\
\hline Device placed in toilets & Sniffer dogs in the station \\
Device planted in rubbish bin & 24/7 CCTV \\
Device planted under seating & Security guards in the station \\
Device planted in ticket office & Search passenger bags \\
Device concealed in luggage & Search passengers \\
Device concealed on attacker's person & Secured access to the ticket office for staff only \\
Device concealed in luggage and placed in lost property & $\begin{array}{c}\text { Place photo booths in discreet corners of the station to } \\
\text { avoid them being a target for exploitation } \\
\text { Device detection system in the toilets }\end{array}$ \\
Device planted in bicycle racks & Reinforced windows on the office front \\
Attacker uses weapon on passengers & Reinforced doors on the front of the station \\
Weapon concealed in attacker's luggage & Reinforced glass on the station windows/doors \\
Device detonated on attacker's person & \\
Weapon concealed on attacker's person & \\
Chemical weapon used & \\
Device placed in photo booth & \\
Device thrown into the station & \\
Explosive filled vehicle & \\
Multiple attackers coordinate attack & \\
\hline
\end{tabular}

Chart 11 depicts that the atriums have extreme, high, and medium risks associated with them. The chance of an extremely severe risk occurring is $47.1 \%$, a highly severe risk occurring is $29.4 \%$, and there is a $23.5 \%$ chance that a medium severity risk will occur. 


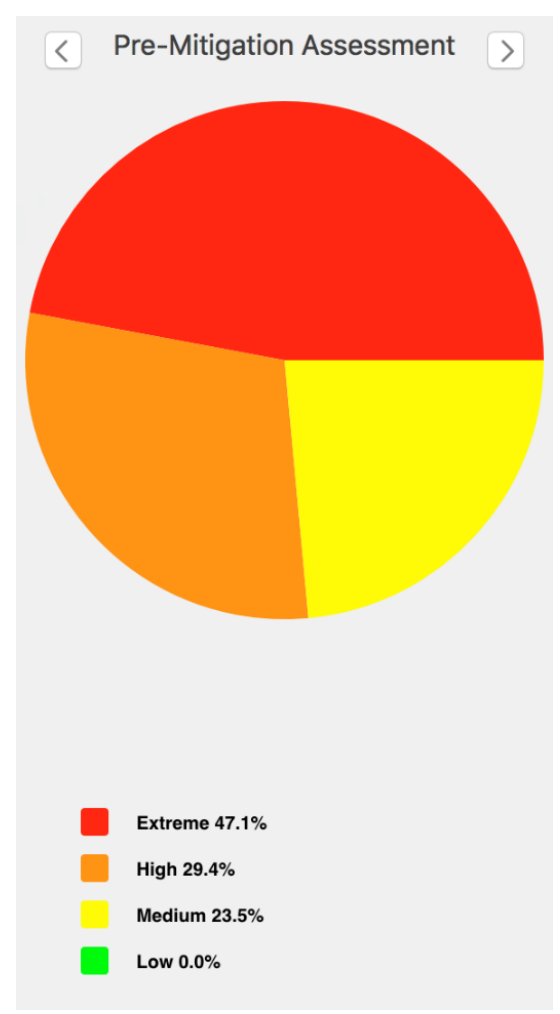

Chart 11. Atrium risk severity pre-mitigation assessment.

Chart 12 depicts that after the mitigation solutions have been theoretically applied, the atriums have high and medium risks associated with them. The chance of a highly severe risk occurring is $47.1 \%$, and there is still a $52.9 \%$ chance that a medium severity risk will occur.

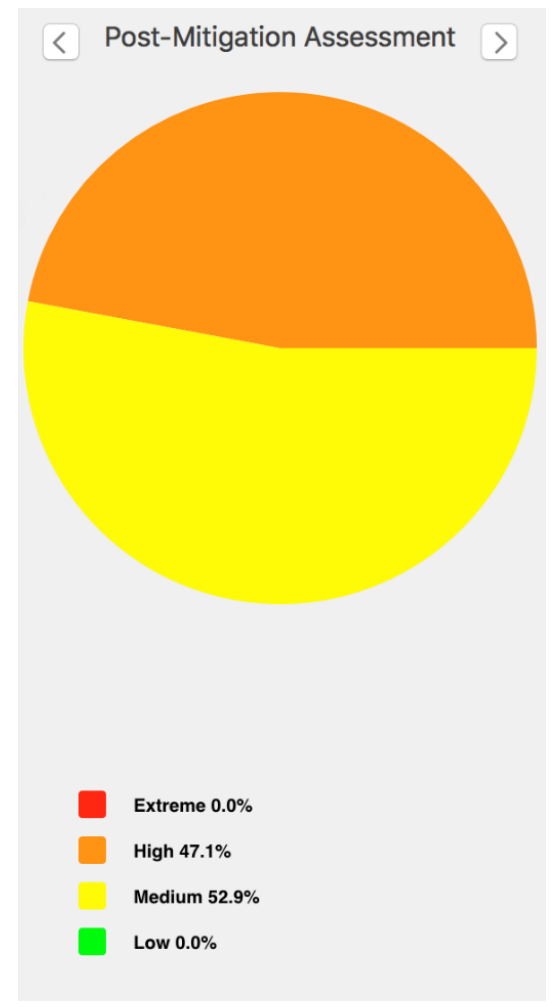

Chart 12. Atrium risk severity post-mitigation assessment. 


\section{Mitigation Strategy Analysis}

- $\quad$ Risk Assessment Severity Charts

It is evident from the post-mitigation assessment risk severity charts that the locations with the highest risk are the shops and restaurants. This is due to the number of additional risks that are associated with the shops and restaurants that are not encountered in all locations of the station. It will also be noted that regardless of the implemented mitigation solutions, the post-mitigation assessment risk severity charts still display significant levels of high severity threats. This is a result of the nature of the risk because if any risk was to evade detection and occur, the effects of the risk would still be devastating, as the mitigation was not done to minimise the impact of the risk, but to prevent it from occurring altogether. This factor must be taken into consideration when the severity charts are analysed. The mitigation strategies applied to the scenarios then evaluated were the strategies that reduced the possibility of an attack occurring, such as security guards being positioned around the station. The strategies that completely eradicated the risk were not applied in the assessment.

- $\quad$ Mitigation Strategies

The strategies proposed for mitigation purposes vary. Some of the suggested strategies are already in use today across train stations, such as the 24/7 CCTV. The use of security guards and sniffer dogs is also something that is widely acknowledged as being good practice, and stations recruit these services fairly often, depending on the station capacity. The idea of searching bags and passengers is one that is adopted in airports internationally as a way of ensuring security and safety for passengers taking to the air, however it is not a practice that has been used in train stations before. The strategies that discuss locking services at night and preventing public access to staff work spaces are, again, something done universally, as is security tagging all merchandise in shops. The removal of station rubbish bins is something that some stations such as the London Underground decide is necessary [30]. However, most stations will decide that the inconvenience this generates is more significant. Preventing track access is becoming increasingly common in underground and metro stations but is yet to be a standard practice for aboveground trains, due to the potentially excessive cost of installing screens along the platform, so passengers cannot reach the tracks. A number of the proposed mitigation strategies require significant effort for little minimisation in risk. These include the ideas of placing the photo booths in discreet corners of the stations, disallowing knives to be stocked in station shops, and providing plastic utensils instead of metal. Whilst other ideas of reinforcing windows and doors across the stations are occasionally already in use as a result of assault from members of the public and not terrorism, they will still serve as a suitable preventative aid.

- Calculations

Based on [2,31], direct method of analysing probability of event occurrence:

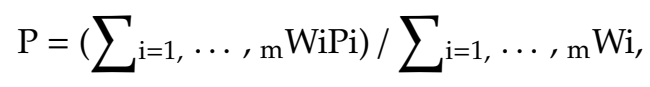

where P: probability; m: narrowly defined samples; i: Wi cases in population of interest; Pi: probability of event in defined sample.

Probability that there will be an attack on the railway in the next five years:

$\%$ increase since 2000: $(1013 / 1651)^{*} 100=61.4 \%$

$\mathrm{P}=\left(0.61^{*} 1651\right)^{*}(1651 / 1826)+[(1826-1651) / 1826]^{*} 0$

$\mathrm{P}=91 \%$ chance there will be an attack on the railway in the next five years.

\section{Optimum Strategy}

\subsection{Aspects of Assessment}

- Population Flow Impact 
The applied mechanism must be capable of providing maximum protection to commuters without impacting the rate at which they progress through the train station. The chosen mitigation solution must also be one that is easy to install, in terms of the minimal disruption it causes to commuters and regular station operations. Additionally, it is crucial the chosen solution be ergonomic, permitting immediate use by the public without extensive explanation or instruction on how to do so. Fast travel with minimal delays is ideal for commuters. If not achieved, it may discourage the use of rail travel.

\section{- Cost Benefit}

The cost of the implemented mitigation solution must relatively equate to the value of the benefit that it will provide. The US government has spent $\$ 300$ billion on counter-terrorism mitigation solutions since 2001 [32]. Although a terrorist mitigation solution is one that will save lives, which are arguably priceless, there is the possibility that the lives will never actually be in direct danger, which would render the mitigation solution itself potentially excessive. In order to calculate if a specific mitigation solution is worth the money it costs to implement it, a number of variables must be taken into account. The net benefit is the indication of whether the mitigation solution will be worth the cost. The variables that determine the net benefit consist of the number and type of threat scenarios, the probability of an attack with and without the mitigation solution in place, the loss or consequence of not having the solution, the number of loss attributes, the cost of the solution, the percentage of risk reduction due to the solution being implemented, and a number of relative probabilities regarding the relative threat, given an attack, and the losses, given the occurrence of a particular threat, without an implemented solution [32]. All of these must be considered when determining how cost-effective a solution will be.

\subsection{Solution Proposal}

The optimum mitigation strategy is a combination of two solutions: to search passengers and to search their luggage. Searching passengers ensures the removal of any equipment that could cause other passengers, staff, or station occupants harm. This process is also simple, easy, and cost-effective as it means that other mitigation methods do not need to be strictly applied to the stations or the trains because the attackers will be apprehended upon entry to the station. This will be discussed further. This mitigation strategy will ensure that people feel safe and secure when travelling. It will require new equipment to be installed at each station across the nation and will therefore demand a fairly considerable initial cost. However, the equipment will eliminate the requisite for other mitigation solutions and thereby the expense of implementing those other solutions. The installation of this mitigation solution will also in essence pay for itself should an attack occur, for the cost of building repair and potential compensation for loss of life or injury would be substantial. The repercussions of an attack also cost the government and the railway companies significantly, for attacks generate fear, which makes people reluctant to travel using that medium. This was evident when an attack on Thalys resulted in a ' $20 \%-30 \%$ loss of ridership' in the subsequent months after the attack [18]. The ergonomic concept will be familiar to many passengers, due the similarity to airport security checks. A result of this will be the ease and pace at which commuters understand and utilise the new mechanisms, minimising disruptions to their journeys, in addition to eradicating further repercussive costs as a result of delays.

A more specific recommendation for an optimum mitigation strategy is a modified version of the Thales Smart Corridor. It should be used as an initial start point or base design, with the existing configuration of desirable capabilities adapted to better suit the requirements of this scenario. Modifications are required to be made before the Smart Corridor's implementation in the nation's train stations as the Corridor is 'approximately three to four metres long', which would be an undesirable extension to all entrances in every station [33]. A combination of multiple technologies, it provides an extremely apt solution to the issues presented regarding a suitable mitigation solution. The existing capabilities include CCTV to count people, detect abandoned objects, and identify suspicious behaviour such as abrupt changes in direction. There is also an incorporated biometric control system that 
identifies passengers by means of facial recognition software. Finally, sensors that can detect explosives, metal and dielectric objects that are concealed beneath clothing are fitted. The Corridor is completely transparent yet manages to fully inspect the passengers as they move through the mechanism, whilst the passengers only experience a 'slight breeze' [34], Sci-fi screening process proposed by Thales, 2008.

\section{Supplementary Considerations}

\subsection{Risk Assessment Analysis}

- $\quad$ Triggers

The risk assessment analysis did not consider the triggers that incentivise terrorist attacks. This is due to the fact that whether it is an individual or a group as part of a larger organisation, research [2] shows there are a multitude of reasons why people would decide to perform an act of terrorism. For example, each political decision will have support and opposition. Radicals, however, may choose to voice their dissent through an attack. Terrorists are not known for their rationality; anything can be a trigger. It is therefore impractical to monitor or attempt to control or reduce apparent triggers.

\section{- $\quad$ Linked Risks}

The analysis did however list the 'linked risks': risks that occur as a result of each other. For example, the risk of a device being planted in a rubbish bin around the station is dependent on the risks of a terrorist either concealing a device on their person or in their luggage. Linked risks are useful in establishing a prioritised list of risk mitigation. See Figure 3.

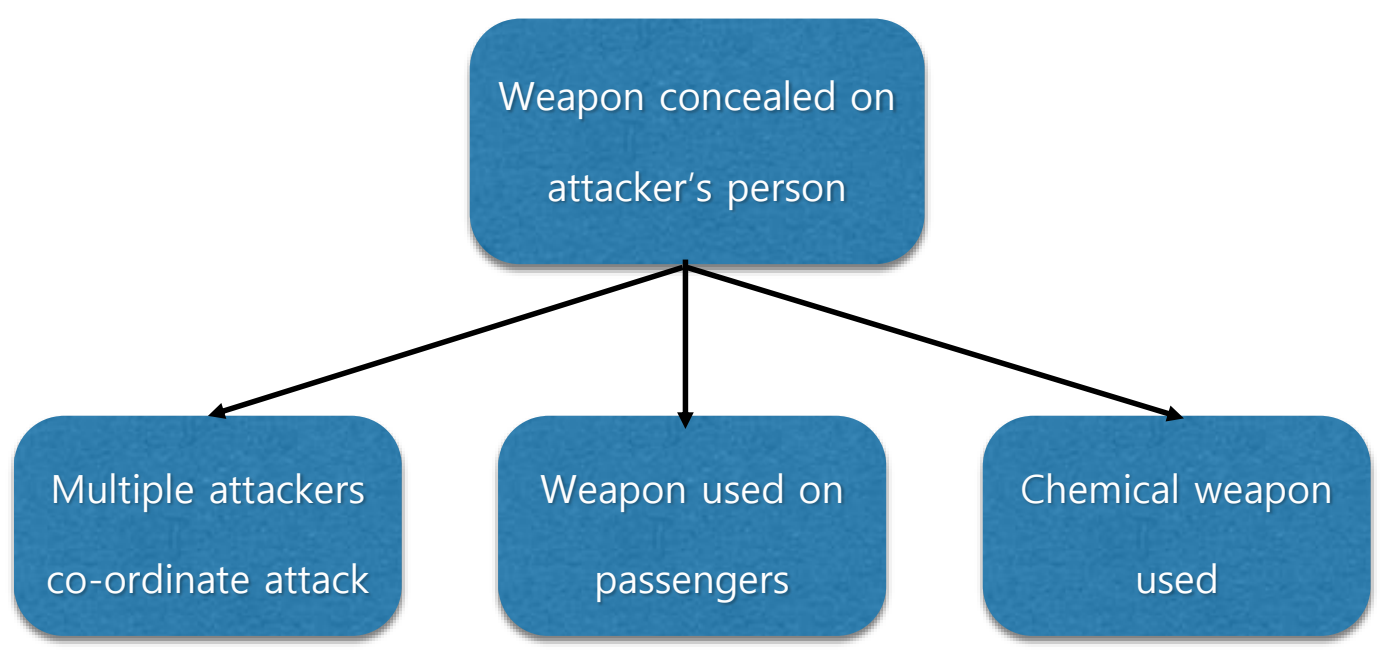

Figure 3. Linked risks of a weapon concealed on an attacker's person.

Linked risks demonstrate the requirement to focus on a workable mitigation solution to eliminate the key risks, so that dependant risks will be subsequently eradicated.

\subsection{Workable Solution Analysis}

- Criminal Treatment

A commuter concern with regards to being searched is being treated like a criminal without providing an reason for such treatment. However, as the analysis has shown, being searched is the most effective mitigation solution and therefore a necessary procedure to ensure that any criminals amongst the populace are identified and removed, so that they are unable to cause harm to those commuters, the majority, who are not criminals.

- Privacy Violation 
The level of detail necessary to produce an image suitable for security inspection has been a topic of controversial debate for some time. Existing scanners are capable of generating images so intricate, it is possible to identify the size of passengers' breasts and genitals [33]. This is an issue that the Thales Smart Corridor does not encounter as it does not create a silhouette of the passenger and uses passive devices in compliance with all health, privacy, and safety regulations [34]. This means all passengers can use the Smart Corridor, or a modified version that harnesses this characteristic, without fear of explicit exposure and under-aged members of the populace can make use of the mechanism without indecent images of minors being produced.

\section{- $\quad$ Necessity}

Despite the fact that acts of terrorism are increasing, they remain fairly rare. When considering the number of unimpeded journeys made by passengers compared to the number disturbed by an act of terrorism, the idea of mitigation methods seems pointless. However, with the predicted climb in passengers and the expansion of the railway network, attacks targeting the railways could become increasingly common [18]. This means that it will become even more vital to have reliable and effective security measures in place to ensure passenger safety. Conducting passenger searches is a simple way to maximise commuter security by preventing attacks, as opposed to working to reduce the aftereffects of an attack and the damage to people and property. Unfortunately, due to the rise in attacks that have occurred on British soil, the remainder of the world could become increasingly fearful of further attacks and hence concerned about their safety should they visit or relocate to the UK. This could have a detrimental effect on British trade and tourism, similar to how Thalys was affected when attacked [18]. Therefore, it is essential that appropriate mitigation solutions are implemented to provide global assurance that the UK is safe.

\section{- Racial Profiling}

Another objection to being searched is the 'random', more extensive searches that occur in airports and the tendency to select a person who fits a specific profile. It is feared that this apparent prejudice could be transferred to railway searches if they are implemented. The public have voiced concerns that racial profiling could become more common, with 'specific parts of the population being targeted' [35]. However, it has been acknowledged that a significant number of attackers do not fit typical racial profiles. This means that they would not be selected for more extensive searches and could potentially avoid detection, whilst innocent civilians who happen to fit a typical profile could be wrongly questioned. The 'shoe bomber' Richard Reid apparently 'did not fit a racial profile' [34] and the attacker who boarded a train in Switzerland with 'fire and a knife' was revealed by police to be 'a Swiss citizen, with a "typical Swiss name"' [36]. Each of these attackers did not fit a typical racial profile. The mitigation mechanism implemented must avoid racial prejudice and treat all passengers with no discrimination and equal treatment.

\section{- Search upon Entry}

Due to attacks such as the 2016 metro and airport bombings in Brussels [36], where a suicide bomber entered the airport terminal in Brussels and detonated his device 'in the departure hall near the check-in desks', it has become clear that an effective mitigation solution is required to check all persons upon entry to the station. Any civilian who can gain access to a train station then has the capacity to detonate a device in the building and cause harm to the infrastructure and surrounding populace, regardless of whether they have purchased a ticket or whether they intend to travel themselves. It is therefore evidently necessary that the search mechanism in place must be positioned at each entrance to the station to prevent this type of attack from occurring again.

- Installation Locations 
Common locations for terrorist exploitation are the more popular train stations in cities, as terrorists capitalise on the population density within the stations to create the greatest impact and attract the most media attention, to achieve their aim of causing the biggest commotion possible. Certain stations in the UK experience an inordinate volume of foot traffic, with the most popular station Waterloo, Greater London, encountering 99,201,604 people annually [37]. This makes Waterloo a prime target for terrorist activity, so naturally there are security measures around the station, however, a passenger can board a train at a more rural location with minimal security measures, such as Shippea Hill, which had only 22 visitors in 2014-2015 [38] and travel into Waterloo, entering the station unimpeded. This highlights the requirement for the mitigation mechanisms to be installed at every station across the country, regardless of how popular they are, to ensure maximum success in mitigating the terrorist threats. Recommended is Thales Smart Corridor, 2017 [39].

\section{Conclusions}

Terrorism is unfortunately becoming more familiar to the world in modern society, with terrorists turning on their own countries in demonstrations of extremism. All the nations need to prepare, as more frequent and increasingly substantial attacks are to come, and work collectively to protect their industry and their people whilst ensuring the necessary capacity to do so. It is evident from this study that acts of terrorism are becoming more common and the expansion of the railways is going to make train and subway stations even greater targets. Fail-safe mitigation strategies are required to assure the continued safety of railway infrastructure and the public. The extensive research and analysis conducted in this study have demonstrated that the most effective mitigation strategy to adopt is to implement a passenger searching mechanism such as a modified version of the Thales Smart Corridor, to efficiently identify and eliminate any suspicious objects and individuals in order to maintain public faith and dependence on the railways for years to come.

\section{Further Research}

The work done throughout this study is a foundation for a concept that could save lives and money. However, it is limited. Should this work be taken further and considered for implementation, additional research must be conducted.

- Software Package

One area that requires significant attention is the software package used to analyse the data collected. Although useful for this report, the Risk Manager software is limited. This software relies on the input of the user to determine the potential risks and in this instance the user simply listed all risks that occurred to them; however, this means that risks faced by the railways could have been overlooked. The nature of risks that are omitted will vary, although it is reasonable to assume that risks of a particularly sinister description will not be envisaged and will consequently not be featured in the analysis. Therefore, the analysis could provide incomplete data from which inaccurate conclusions are drawn. Existing station risk assessments should be examined and those featured risks should all be entered into the software for analysis.

Within the Risk Manager software, each risk is categorised by the likelihood of the event occurring and the severity of the risk, should it occur. However, both characteristics are subjective, with both the likelihood and severity of a risk being dependant on multiple external variables each. Therefore, due to the basis of the risk analysis being subjective, the conclusions drawn from the data are circumstantial. In order to rectify this, the risks need to be categorised in a more qualitative manner.

Furthermore, through this software, the analysis performed does not have an impact on the likelihood of an event occurring. The mitigation strategies applied to the risks only result in a reduction of the severity level of the risk, and the likelihood of the event remains the same. This effectively renders the categorisation of risks through their likelihood of occurrence as meaningless. However, the likelihood of a devastating risk occurring is significant when considering the potential impact 
the risk could have on peoples' lives and public infrastructure. Hence, it is imperative any software used demonstrates that when the mitigation solutions are applied to the risks, the consequent analysis reflects an impact on this vital consideration.

\section{- $\quad$ Additional Aspects}

Station Flow analysis should be undertaken once the chosen mitigation solution has been implemented. This is to obtain further and legitimate validation that this solution is the optimum, works in real time, and does not have a significant negative impact on public usage of the station and its facilities.

The understanding that the execution of mitigation solutions will result in the reduction or elimination of risks encountered by the railways should be substantiated through deeper analysis of the linked risks. A risk identified as being the cause of another risk should be scrutinised individually and extensively to ensure that the application of the proposed mitigation solution will render the risk, and therefore all linked or associated risks, non-existent.

Author Contributions: A.L. developed the initial draft of the paper. M.M. formulated the aims and the scope of this work. M.M. was instrumental for editing, reviewing and introducing quality and continuous improvements. All authors have read and agreed to the published version of the manuscript.

Funding: This research received no external funding.

Conflicts of Interest: The authors declare no conflict of interest.

\section{References}

1. Global Terrorism Database ${ }^{1}$. GTD Search Results. 2017. Available online: https://www.start.umd.edu/gtd/ search/Results.aspx?start_yearonly=2000\&end_yearonly=2001\&start_year=\&start_month=\&start_day= \&end_year=\&end_month=\&end_day $=\&$ region $=1 \&$ country $=217 \&$ perpetrator $=20029 \&$ dtp $2=$ all\&success $=$ yes\&casualties_type $=b \& c a s u a l t i e s \_m a x=($ accessed on 11 May 2017).

2. Global Terrorism Database ${ }^{2}$. GTD Search Results. 2017. Available online: https://www.start.umd.edu/gtd/ search/Results.aspx?start_yearonly=1970\&end_yearonly=2015\&start_year=\&start_month=\&start_day= \&end_year=\&end_month=\&end_day=\&region=12\&region=2\&region=7\&region=4\&region=9\&region= 10\&region $=1$ \&region $=3 \&$ region $=6 \&$ region $=5 \&$ region $=11 \&$ region $=8 \&$ asmSelect $0=\&$ asmSelect $1=\& \mathrm{dtp} 2=$ all\&success $=$ yes\&casualties_type=b\&casualties_max $=($ accessed on 11 May 2017).

3. BBC News ${ }^{1}$. London Attack: What We Know So Far-BBC News. 2017. Available online: http://www.bbc. co.uk/news/uk-39355108 (accessed on 12 May 2017).

4. Marrone, S.; Nardone, R.; Tedesco, A.; D'Amore, P.; Vittorini, V.; Setola, R.; De Cillis, F.; Mazzocca, N. Vulnerability modeling and analysis for critical infrastructure protection applications. Int. J. Crit. Infrastruct. Prot. 2013, 6, 217-227. [CrossRef]

5. MIT Sloan School of Management. MIT Sloan Faculty Expertise Guide: Faculty Bio: Arnold Barnett. 2017. Available online: http://mitsloan.mit.edu/expertiseguide/facultybio.html?w=41132 (accessed on 12 May 2017).

6. Parfitt, T. Train Passengers 'FOUR TIMES More Likely to be Hit by Terror Attack than Fliers'. The Daily Express. 2017. Available online: http://www.express.co.uk/news/world/600042/train-Paris-AmsterdamFrance-terror-attack-plane-public-transport-Arnold-Barnett (accessed on 12 May 2017).

7. Railway Technology. Terrorism: The Ever Evolving Threat. 2010. Available online: http://www.railwaytechnology.com/features/feature88006/ (accessed on 13 May 2017).

8. News.bbc.co.uk. BBC NEWS | In Depth. 2017. Available online: http://news.bbc.co.uk/1/shared/spl/hi/ guides/457000/457031/html/default.stm (accessed on 15 May 2017).

9. Abbott, D.; Marinov, M. An event based simulation model to evaluate the design of a rail interchange yard, which provides service to high speed and conventional railways. Simul. Model. Pract. Theory 2015, 52, 15-39. [CrossRef]

10. Dhir, S.; Marinov, M.; Worsley, D. Application of the analytic hierarchy process to identify the most suitable manufacturer of rail vehicles for High Speed 2. Case Stud. Transp. Policy 2015, 3, 431-448. [CrossRef] 
11. Shvetsov, A.V.; Shvetsova, S.V. Protection of high-speed trains against bomb-carrying unmanned aerial vehicles. J. Transp. Secur. 2017, 10, 115-126. [CrossRef]

12. Brice, D.; Marinov, M.; Rueger, B. A Newly Designed Baggage Transfer System Implemented Using Event-Based Simulations. Urban Rail Transit 2015, 1, 194-214. [CrossRef]

13. Yeung, H.K.; Marinov, M. A systems design study introducing a collection point for baggage transfer services at a railway station in the UK. Urban Rail Transit 2019, 5, 80-103. [CrossRef]

14. Toal, J.; Marinov, M. A check-in and bag drop service onboard light rail vehicles for passengers travelling to the airport. Sustain. Rail Transp. 2019, 3, 1-82, (Lecture Notes in Mobility).

15. Dampier, A.; Marinov, M. A study of the feasibility and potential implementation of metro-based freight transportation in newcastle upon tyne. Urban Rail Transit 2015, 1, 164-182. [CrossRef]

16. Kelly, J.; Marinov, M. Innovative interior designs for urban freight distribution using light rail systems. Urban Rail Transit 2017, 3, 238-254. [CrossRef]

17. Potti, P.; Marinov, M.; Sweeney, E. A simulation study on the potential of moving urban freight by a cross-city railway line. Sustainability 2019, 11, 6088. [CrossRef]

18. Leboeuf, M. High-speed rail: Opportunities and threats. Engineering 2016, 2, 402-408. [CrossRef]

19. De Cillis, F.; De Maggio, M.C.; Pragliola, C.; Setola, R. Analysis of criminal and terrorist related episodes in railway infrastructure scenarios. Homel. Secur. Emerg. Manag. 2013, 10, 1-30. [CrossRef]

20. Marinov, M.; Sahin, I.; Ricci, S.; Vasic-Franklin, G. Railway operations, time-tabling and control. Res. Transp. Econ. 2013, 41, 59-75. [CrossRef]

21. Potti, P.; Marinov, M. Evaluation of Actual Timetables and Utilization Levels of West Midlands Metro Using Event-Based Simulations. Urban Rail Transit 2020, 6, 28-41. [CrossRef]

22. Shvetsov, A.V.; Shvetsova, S.V. Research of a problem of terrorist attacks in the metro (subway, U-Bahn, underground, MRT, rapid transit, metrorail). Eur. J. Secur. Res. 2017, 2, 131-145. [CrossRef]

23. Strandh, V. Exploring vulnerabilities in preparedness-rail bound traffic and terrorist attacks. J. Transp. Secur. 2017, 10, 45-62. [CrossRef]

24. Yolmeh, A.; Baykal-Gürsoy, M. Urban rail patrolling: A game theoretic approach. J. Transp. Secur. 2018, 11, 23-40. [CrossRef]

25. Avci, O.; Ozbulut, O. Threat and vulnerability risk assessment for existing subway stations: A simplified approach. Case Stud. Transp. Policy 2018, 6, 663-673. [CrossRef]

26. Kaewunruen, S.; Alawad, H.; Cotruta, S. A decision framework for managing the risk of terrorist threats at rail stations interconnected with airports. Safety 2018, 4, 36. [CrossRef]

27. Shafieezadeh, A.; Cha, E.J.; Ellingwood, B.R. A decision framework for managing risk to airports from terrorist attack. Risk Anal. 2014, 35, 292-306. [CrossRef]

28. RAMPART Project. The Project-RAMPART Project. 2017. Available online: http://www.rampart-project.eu/ project (accessed on 14 May 2017).

29. Matsika, E.; O’Neill, C.; Battista, U.; Khosravi, M.; Laporte, A.; Munoz, E. Development of risk assessment specifications for analysing terrorist attacks vulnerability on metro and light rail systems. Transp. Res. Procedia 2016, 14, 1345-1354. [CrossRef]

30. Telegraph.co.uk. Bomb-Proof Bins to be Installed in London. 2017. Available online: http://www.telegraph. co.uk/news/newstopics/howaboutthat/3327528/Bomb-proof-bins-to-be-installed-in-London.html (accessed on 15 May 2017).

31. Alemi, F.; Sinkule, J. Security risk analysis. In Decision Analysis for Healthcare Managers; Chapter 9; Health Administration Press: Ann Arbor, MI, USA, 2006; ISBN 13 978-1-56793-256-0.

32. Stewart, M. Risk-informed decision support for assessing the costs and benefits of counter-terrorism protective measures for infrastructure. Int. J. Crit. Infrastruct. Prot. 2010, 3, 29-40. [CrossRef]

33. Boussadia, K. The evolution of airport screening technology. Biom. Technol. Today 2009, 17, 7-8. [CrossRef]

34. Thales, Sci-fi screening process. Biom. Technol. Today 2008, 16, 3-4. [CrossRef]

35. Paraskevas, A.; Arendell, B. A strategic framework for terrorism prevention and mitigation in tourism destinations. Tour. Manag. 2007, 28, 1560-1573. [CrossRef]

36. Adams, S.; Milanian, K. Swiss Train Passengers Attacked with 'Fire and Knife' Sparking Terror Fears. The Mirror. 2017. Available online: http://www.mirror.co.uk/news/world-news/terror-swiss-trainpassengers-attacked-8624527 (accessed on 13 May 2017). 
37. Deorden, L. Brussels Terror Attacks: At Least 30 Dead After Suicide Bombing and Explosions at Airport and Metro Station. Idependent.co.uk. 2016. Available online: http://www.idependent.co.uk/news/world/ europe/several-wounded-after-two-loud-explosions-heard-at-brussels-airport-a6945381.html (accessed on 13 May 2017).

38. BBC News2. Revealed: Britain's busiest and quietest stations-BBC News. 2017. Available online: http://www.bbc.co.uk/news/uk-35105105 (accessed on 13 May 2017).

39. Thales, Thales Smart Corridor. 2017. Available online: https://www.thalesgroup.com/fr/worldwide/securite/ press-release-54 (accessed on 12 May 2017).

(C) 2020 by the authors. Licensee MDPI, Basel, Switzerland. This article is an open access article distributed under the terms and conditions of the Creative Commons Attribution (CC BY) license (http://creativecommons.org/licenses/by/4.0/). 